\title{
Measuring Profitability From the Use of Personal Data For Targeted Ads
}

\author{
Aidan Katsikas ${ }^{1}$ and Vimal Murugan ${ }^{2}$ \\ Warrington College of Business ${ }^{1}$, College Liberal Arts and Sciences ${ }^{2}$
}

Faculty mentor: Anne Donnelly, Center for Undergraduate Research

\begin{abstract}
Companies like Facebook and Google track their users' data to enhance their ads and offer highly targeted ad space. The question is then raised: how much more profitable does collecting data make companies and what is the future of these companies, as rising data privacy concerns are believed to result in the passage of future legislation? In order to isolate the effects of collecting data on profit, the revenue and revenue growth of Google and Facebook were compared to their competitors that do not collect data and revenue was adjusted to find the average per user. By adjusting each company's revenue for its number of users, Google and Facebook could both be effectively compared to their significantly smaller competitors. Google was compared to another search engine, DuckDuckGo, and Facebook was compared to a social media site, Yubo. Our analysis found that both Google and Facebook earned significantly larger amounts of revenue, per user, than their competitors who do not collect data. However, DuckDuckGo and Yubo both experienced considerably larger revenue growth this past year, highlighting improved success among companies relying on different models for generating revenue. Our research and similar studies will become more important once legislation is passed, as companies may have to pay taxes or fees associated with acquiring users' data. Regardless of what new regulations entail, it will be essential to quantify the profitability of collecting users' data.
\end{abstract}

\section{Introduction}

\section{Literature Review}

It is a common misconception that our electronic devices are "listening to us." When an advertisement appears for the exact item someone is looking to buy, this may be the only explanation they can render. While our phones cannot actually hear what we say, the reality is not too different. Using a system that one study refers to as "surveillance capitalism," companies like Google and Facebook collect data from their users' actions and use that to offer buyers effective ad space (Deibert, 2019). After recording what users view and search, the companies 
use algorithms to predict who particular ads will prove most effective for (Deibert, 2019). Another study explains that over time, those accumulating personal data can develop profiles for specific people; these profiles provide insight into potential transactions individuals are looking to make in the future (Yeh, 2018).

Data collection has essentially brought on a new age of advertising. Companies can now pay for their ads to be shown to individuals that are more likely to be interested in their products. This newer form of advertising is empirically proven to be more effective than traditional forms (Ghose, 2016). Understandably, businesses want to spend their money as effectively as possible on advertising. This process has made personal data a valuable commodity and created a billiondollar industry. Not only is this industry extremely profitable, but the dominance of social media and the internet in modern society have cemented the data market as a durable one. While much of the United States economy has suffered from the effects of the Covid-19 pandemic, data companies have actually seen an increase in revenue. In a recent study, mathematical models suggest that the U.S. economy could shrink by over $30 \%$ from the beginning of the pandemic by the time vaccines are effectively able to end the pandemic (Goldsztejn, 2020). Meanwhile, companies including Facebook and Google yielded large increases in revenue compared to last year (MarketLine, 2021). This comes at little surprise, considering that advertising accounts for 98.5\% of Facebook's revenue (Statista, 2020). Media sites have created a system in which advertisers are the consumers and users are the product - and this system consistently earns money.

The targeted advertising model has proven it can withstand drastic changes to the economy. However, one potential obstacle does present a threat to the profitability of the industry: data privacy. As of now, data collection is relatively unregulated in the United States. This presents ethical problems as companies can extensively record people's personal data, selling it without their knowledge (Birckan et al., 2020). Data Analytics in a Privacy Concerned explains how the European Union has already passed legislation to deal with this ethical dilemma. The General Data Protection Regulation (GDPR) requires companies collecting personal data to adhere to specific measures to ensure citizens' privacy (Wieringa et al., 2021). Many speculate that similar legislation will soon be passed in the United States. Measures to regulate the buying and selling of data, designed to promote privacy, can actually limit the profit earned by those buying and selling data. Some have begun to advocate for personal ownership of 
one's data. In this case, everyone would own their own data, reserving the right to sell it if they wish. Not only would this tackle the issue of data privacy, but it would also create a balance in the data industry; data companies would have less ability to influence consumer actions (Saleh Bataineh et al., 2020).

Before exploring what the future of the personal data market will look like, an important question must be answered: how much profitability does personal data bring to companies that utilize it? Placing a value on the worth of our data is essential to understanding the industry both now and in the future. According to researcher Chris Berg, the data surveillance industry is poised for change; stricter regulation and a public push for more privacy will likely be key factors in this change (2019). Placing an estimate on the monetary value of data would allow us to analyze the impending changes in the industry and display a potential causal relationship between the use of personal data and company output. Google and Facebook — despite offering users very different experiences - essentially offer the same services to advertisers (Pekala, 2017). Both companies track users' data over time, allowing them to offer their customers effective, targeted ad space. Google's largest competitor that does not track user data is by far DuckDuckGo; while the company relies on advertising to generate revenue, ads are displayed to users solely based on their search query (Norem, 2017). An identical search will yield the same response for different users, as these sites do not collect and store personal data. Rising privacy concerns in recent years has allowed services such as DuckDuckGo to make staggering growth. The number of queries DuckDuckGo received more than doubled from 2018 to 2020 going from a total of over 9 billion in 2018 to over 23 billion in 2020 (DuckDuckGo Traffic). This growth is significant, but DuckDuckGo still only owned $0.64 \%$ of the total search market share in 2020 while Google dominated with a share of $92.54 \%$ (Statista). There is still a lot that is unknown about how much exactly collecting data aids these businesses and how much value they gain from collecting your personal data. Concerns over privacy and legislation may potentially diminish these companies' ability to profit in the future. With these concerns it is important to understand the value and impact of this data on these companies.

Data collection has completely revolutionized marketing and how companies interact with consumers, and the industry also has the potential to exponentially grow. Learning precisely how much value is added to businesses that use data collection has its obvious merits for those operating a business, but it has philosophical implications as well. Many ethical concerns 
regarding privacy have been arising in regard to data collection and understanding how much your privacy and data is essentially worth, can influence your decision making as a consumer. With many calling to change public policy and legislation, this information can be useful to lawmakers as well.

Ethical concerns are likely to drive legislative changes in the United States, especially in the near future (Birckan, 2020). In order to pass comprehensive legislation, it is essential to understand the impact that changes within the industry will have on some of the largest companies in human history. The issue of data privacy has increasingly gained more attention in recent years; further regulation of this industry will serve as one of the greatest examples of government regulation in modern American history. Understanding the profitability of the data used by these companies can ensure for a better transition to shift how internet companies collect and value data.

\section{Design and Methods}

For the purpose of this study, we will examine the value of data used by both Google and Facebook. Google and Facebook were chosen because they represent the largest internet browsing and social media services, respectively. Additionally, both companies spearheaded the current model of targeted advertising in order to generate enormous revenues (Lee, 2011). In order to estimate the value of user data we decided to compare Google and Facebook with their largest competitors that do not track user data. DuckDuckGo was selected for comparison to Google, as it essentially offers the same services, but without targeted advertising. While the privacy-focused search engine still generates revenue through ads, it "does not collect, store, track, or share any personal information about you" (Cross, 2019). Moreover, Yubo, a rapidly growing social media site served as a relatively prominent competitor of Facebook, which also does not track users' data. Yubo collects no personal information, as it relies exclusively on a subscription-based model for generating revenue.

To compare the two categories of electronic media companies - those collecting personal data and those that are not not - different statistics were compared, providing insight into the profitability of each companies' revenue generation model. Of course, since both Google and Facebook are significantly larger than DuckDuckGo or Yubo, all measurement figures were adjusted for the number of users. This eliminates the largest confounding variable in comparing 
the profitability of the different companies' business models. The purpose of this study is to compare the overall success of companies tracking user data, and those not doing so. Any difference in company performance statistics provides insight into the true value of personal data for Google and Facebook.

This study compared the revenue each company brings in, adjusted for the number of users each site has. Since different sites vary in size, adjusting for the number of users each site has was a necessary step for comparison. Revenue divided by the number of users revealed how much each company makes per user. Comparing this figure for Google and Facebook to Yubo and DuckDuckGo allows us to better understand the worth of utilizing personal data.

Examining each company's revenue growth rate provided insight into how quickly each company is growing. Google and Facebook's dominance of their respective markets will likely contribute to a steady growth, especially with an increase in the popularity of these platforms during the COVID-19 pandemic. However, an increase in support for increased data privacy has brought many new users to alternative sites, like DuckDuckGo and Yubo. By recording a measurement for revenue growth, we learned how trends with the popularity of varying platforms translate to the amount of revenue they bring in.

By comparing Google and Facebook to their competitors, who do not collect their users' data for targeted advertising, the true value of this data can be realized. The research hypothesized that tracking users' personal data led to an increase in profitability; targeted advertisements have been proven to be more effective and should allow companies using this method to bring in more revenue from customers. Alternatively, companies that do not collect data from their users to experience greater revenue growth rates, as evidence shows that sites prioritizing data privacy are increasing in popularity. However, Google and Facebook also could have seen larger-thanusual revenue growth, as both companies adapted well to the Covid-19 pandemic. The analysis of all four companies' profitability will be examined in the results section.

\section{Results}

Google's ad revenue in 2020 was $\$ 146.92$ billion with 4.258 billion users, and they had a revenue growth of $12.77 \%$. Facebook's revenue in 2020 was $\$ 85.965$ billion with 1.845 billion users, and they had a revenue growth of 21.6\%. DuckDuckGo's revenue in 2020 was \$25 million 
with 25 million users, and they had a revenue growth of 30\%. Yubo's revenue in 2020 was $\$ 20$ million with 30 million users, and they had a revenue growth of $100 \%$. After adjusting the revenue for the number of users, Google had a revenue of $\$ 36.70 /$ user, Facebook had a revenue $\$ 46.59 /$ user, DuckDuckGo had a revenue of $\$ 1 /$ user, and Yubo had a revenue of $\$ 0.67 /$ user. Google made 36.7 times more per user than DuckDuckGo, however, Google's revenue growth was $12.77 \%$ while DuckDuckGo's revenue growth was 30\%. Facebook made 69.5 times more per user than Yubo, however, Facebook's revenue growth was 21.6\% while Yubo's revenue growth was $100 \%$.

Table 1. Companies' Revenue and Number of Users

\begin{tabular}{|l|l|l|}
\hline & Revenue & Users \\
\hline Google & $\$ 146.92$ billion & 4.258 billion \\
\hline Facebook & $\$ 85.965$ billion & 1.845 billion \\
\hline DuckDuckGo & $\$ 25$ million & 25 million \\
\hline Yubo & & 30 million users \\
\hline
\end{tabular}

Table 1. Company Revenue per User and Revenue Growth

\begin{tabular}{|l|l|l|}
\hline & Revenue per User & Revenue Growth \\
\hline Google & $\$ 36.70 /$ user & $12.77 \%$ \\
\hline Facebook & $\$ 46.59 /$ user & $21.6 \%$ \\
\hline DuckDuckGo & $\$ 1 /$ user & $30 \%$ \\
\hline
\end{tabular}




\begin{tabular}{|l|l|l|}
\hline Yubo & $\$ 0.67 /$ user & $100 \%$ \\
\hline
\end{tabular}

\section{Conclusion}

The study's results support the original hypothesis. After adjusting each companies' net income for the number of users on their site, both Google and Facebook held a significantly larger revenue per user than DuckDuckGo and Yubo. This finding suggests that revenue generation models which rely on collecting users' data for targeted advertising led to increased profitability compared to those who do not. Since internet sites charge advertisers only for ads that are interacted with by users, it makes sense that a highly targeted method of advertising would lead to increased revenue (Lee, 2011). Any legislation regulating the collection of data would almost certainly reduce the amount of revenue that Google and Facebook earn. In this case, DuckDuckGo would not be impacted as they do not collect any data. It is important to note that Google and Facebook collecting more revenue per user can also be partially attributed to the greater popularity and resources that both companies have compared to their competitors. However, by adjusting the revenue generating for the number of users each site has, we believe that this confounding variable was mostly eliminated.

The results also show that DuckDuckGo and Yubo are growing at a greater rate than Google and Facebook. This can be attributed to the growing popularity of data privacy in the United States. DuckDuckGo and Yubo are also younger companies, which are more likely to grow at a faster rate than more established companies, like Google and Facebook. Over time, this trend might lead to greater profitability for revenue generation models that do not rely on the collection of personal data.

Going forward, companies like Google and Facebook will benefit from exploring other methods of bringing in revenue - especially as legislation in the United States becomes more likely to be passed. With the collection of their users' personal data more heavily regulated, continuing to rely on their current systems will result in decreases in profitability among these companies. 


\section{Acknowledgements}

We would like to thank our student mentor, Max Mathers, as well as our faculty members Dr. Anne Donnelly and Professor Michelle Leonard for their vital contributions to our research. We would also like to thank the Undergraduate Research Scholars Program for granting us the opportunity to conduct this research.

\section{References}

Berg, C. (2019). Regulate? Innovate! [Article]. IPA Review, 71(2), 32-39.

Birckan, G., Dutra, M., Macedo, D. d., \& Viera, A. (2020). Effects of data protection laws on data brokerage businesses [article]. EAI Endorsed Transactions on Scalable Information Systems, 7(27). https://doi.org/10.4108/eai.22-7-2020.165673

Cross, J. (2019). Should Apple have its own search engine? In (Vol. 36, pp. 96-99): IDG Communications, Inc.

Deibert, R. J. (2019). Three Painful Truths About Social Media [Article]. Journal of Democracy, 30(1), 25-39.

Facebook. (January 28, 2021). Facebook's advertising revenue worldwide from 2009 to 2020 (in million U.S. dollars) [Graph]. In Statista. Retrieved April 14, 2021, from https://www.statista.com/statistics/271258/facebooks-advertising-revenue-worldwide/

Ghose, A., \& Todri-Adamopoulos, V. (2016). toward a digital attribution model: measuring the impact of display advertising on online consumer behavior [Article]. MIS Quarterly, 40(4), 889-A830.

Goldsztejn, U., Schwartzman, D., \& Nehorai, A. (2020). Public policy and economic dynamics of COVID-19 spread: A mathematical modeling study [Article]. PLoS ONE, 15(12), 116. https://doi.org/10.1371/journal.pone.0244174

Lee, M. (2011). Google ads and the blindspot debate [Article]. Media, Culture \& Society, 33(3), 433447. https://doi.org/10.1177/0163443710394902

Norem, J. (2017). 4 ways to bolster your online privacy [Article]. PCWorld, 35(5), 205-208.

Pekala, S. (2017). Privacy and User Experience in 21st Century Library Discovery [Article]. Information Technology \& Libraries, 36(2), 48-58. https://doi.org/10.6017/ital.v36i2.9817

Saleh Bataineh, A., Mizouni, R., Bentahar, J., \& El Barachi, M. (2020). Toward monetizing personal data: A two-sided market analysis,. In (Vol. 111, pp. 435-459). Future Generation Computer Systems.

StatCounter. (March 9, 2021). Search engine market share held by DuckDuckGo from July 2019 to February 2021, by region [Graph]. In Statista. Retrieved April 14, 2021, from https://www.statista.com/statistics/1220046/duckduckgo-search-engine-market-share-by-region/ 
Wieringa, J., Kannan, P. K., Ma, X., Reutterer, T., Risselada, H., \& Skiera, B. (2021). Data analytics in a privacy-concerned world [Article]. Journal of Business Research, 122, 915-

925. https://doi.org/10.1016/j.jbusres.2019.05.005

Yeh, C.-L. (2018). Pursuing consumer empowerment in the age of big data: A comprehensive regulatory framework for data brokers. In (Vol. 42). Telecommunications Policy. 